\title{
Recomendaciones para la atención odontológica según el riesgo de infección por COVID-19
}

\section{DOI 10.5377/alerta.v4i2.9650}

Wendy Yesenia Escobar de González ${ }^{1}$, Guillermo Alfonso Aguirre Escobar ${ }^{2}$, Katleen Argentina Aguirre de Rodríguez $^{3}$, Francisco José Rivas Cartagena ${ }^{4}$, Ester Abigail Pérez Rodas ${ }^{5}$, Ana Lourdes Pérez Siciliano ${ }^{6^{*}}$

1-6 Universidad de El Salvador, San Salvador.

${ }^{*}$ Correspondencia

$\checkmark$ lourdes.siciliano@ues.edu.sv

1. (D) 0000-0001-9725-0783

2. (1D) $0000-0003-0188-6379$

3. (1) 0000-0002-9200-4214

4. (1) $0000-0002-9223-8088$

5. (1) 0000-0002-0827-9355

6. (1) 0000-0002-6470-0933

Main recommendations for the care of dental emergencies according to the risk of infection by COVID-19

\section{Citación recomendada:}

Escobar de González WY, Aguirre Escobar GA, Aguirre de Rodríguez KA, José

Rivas Cartagena FJ, Pérez Rodas EA, Pérez Siciliano AL. Recomendaciones para la atención odontológica según el riesgo de infección por COVID-19. Alerta. 2021;4(2):5460. DOI: 10.5377/alerta. v4i2.9650

\section{Recibido:}

15 de junio de 2020

\section{Aceptado:}

21 de enero de 2021

\section{Publicado:}

21 de mayo de 2021

\section{Contribución de autoría:} WEYG', GAAE ${ }^{2}$, KAAR ${ }^{3}$, FJRC ${ }^{4}$, EAPR ${ }^{5}$, ALPS ${ }^{6}$ : Revisión bibliográfica, análisis y discusión de los datos.

\section{Conflicto de intereses:}

Los autores declaran que no tienen ningún interés comercial o asociativo que presente un conflicto de intereses con el trabajo presentado.

\begin{abstract}
Resumen
EI SARS-CoV-2 es el séptimo betacoronavirus que infecta a la especie humana. Este se caracteriza por una alta transmisibilidad y relativa baja letalidad. La enfermedad se manifiesta, en la mayor parte de la población, de forma leve y aumenta la probabilidad de severidad y letalidad en adultos mayores de 65 años, con enfermedades crónicas no trasmisibles previas, como diabetes, hipertensión arterial, enfermedades respiratorias y obesidad. En este artículo de revisión se presentan parámetros con base en la evidencia científica, que guíen al odontólogo en la toma de decisiones, para la atención odontológica y limitar el riesgo de infección cruzada en el marco del estado actual de pandemia por COVID-19. La llamada o video llamada constituye la primera elección para realizar el triaje, previo a asignar una cita, ya que permite clasificar el riesgo de infección por COVID-19 de un paciente y establecer el nivel de prioridad de tratamiento. El algoritmo para la toma de decisiones en clínicas odontológicas durante el período de emergencia por COVID-19, debe constituirse en una recomendación que los odontólogos que atienden pacientes pongan en práctica. Por cuanto, permite clasificar el riesgo de infección y letalidad del paciente, definiendo los tratamientos en electivos, de urgencia o emergencia.
\end{abstract}

Palabras clave

Infecciones por coronavirus, clínicas odontológicas, contención de riesgos biológicos, toma de decisiones, pandemia. 


\section{Introducción}

La enfermedad conocida como COVID-19, causada por el SARS-CoV-2 fue detectada por primera vez en Wuhan, Hubei, China, el pasado diciembre de 2019. Se ha definido como una enfermedad de alto riesgo para la salud pública a nivel mundial',2. Por ello, la Organización Mundial de la Salud (OMS) declaró el 30 de enero de 2020 que el brote por COVID-19 constituía una Emergencia de Salud Pública de Preocupación Internacional (PHEIC) 2,3. El 11 de marzo de 2020, cuando el virus estaba presente en 114 países, fue declarada pandemia por la OMS4. Según los expertos en virología de la Universidad Johns Hopkins, Estados Unidos, el nuevo coronavirus vino para quedarse; probablemente causará brotes anuales, en su mayoría, casos leves ${ }^{5}$.

Posterior a que la OMS declarara pandemia por SARS-CoV-2, El Salvador decretó estado de emergencia nacional el 11 de marzo. Luego, el 16 de marzo, se tomaron nuevas disposiciones para contener la pandemia, entre las que incluye la suspensión de consultas odontológicas, privadas y públicas, que no sean emergencia ${ }^{6}$. Para dar seguimiento al estado de la pandemia en el territorio nacional, el Gobierno de El Salvador habilitó una página oficial. Según datos procedentes de dicha fuente, hasta el 15 de febrero de 2021, a las 12:05 horas, existían 58023 casos confirmados; 3585 casos activos, 1750 fallecidos y 52688 recuperados por COVID-197.

Esta revisión ha sido elaborada a partir de una amplia revisión de la evidencia científica disponible a la fecha, con la metodología de trabajo con base en riesgo y el sistema de algoritmos. Con el propósito de apoyar al cirujano dental en la toma de decisiones conducentes a resolver urgencias/emergencias de origen bucal, de acuerdo con las disposiciones gubernamentales promulgadas para limitar la expansión de la pandemia en el país. En odontología, por la necesidad de trabajar en contacto cercano con los pacientes debido al área de trabajo, es importante el cumplimiento estricto de los protocolos y barreras de bioseguridad, ya que similar a otros virus, el SARS-CoV-2 representa un riesgo de infección cruzada en la consulta odontológica, principalmente por la generación de aerosoles².

\section{Discusión de la temática}

Los coronavirus (CoV) (orden Nidovirales, familia Coronaviridae, subfamilia Coronavirinae) son virus envueltos con un genoma de ARN monocatenario de sentido positivo. Con ta- maños de genoma de 26 a 32 kilobases (kb) de longitud, los CoV tienen los genomas más grandes para virus de ARN. De manera que, al ser genomas grandes y pesados, su transmisión a través de la emisión de gotículas de fluidos se facilita a cortas distancias ${ }^{8-10}$.

Se ha informado que el período de incubación asintomático para individuos infectados por COVID-19 tiene un promedio de 5, 6 o hasta 14 días ${ }^{11,12}$. Por lo que es la duración internacionalmente recomendada para la observación médica y la cuarentena de personas potencialmente expuestas ${ }^{8}$.

Diversa evidencia científica establece que las enfermedades infecciosas pueden ser potencialmente transmitidas en el ejercicio de la profesión odontológica, tanto a pacientes como a profesionales ${ }^{13}$. Todas las enfermedades virales o infecciosas representan peligro para el odontólogo. Según datos brindados por la OMS, existen diversas enfermedades que tienen tasas de mortalidad similares a las del COVID-19 y que siempre han representado un peligro en la práctica odontológica. Entre estos, el VIH/ SIDA, con una tasa de letalidad del 3,1\%; el virus del H1N1, que presenta una tasa de letalidad del 2 a $3 \%$, afectando en mayor medida a adultos jóvenes. De igual manera, el brote del Síndrome Respiratorio Agudo Severo (SARS-CoV) en los años 2002 y 2003, en la provincia de Guangdong, China. Más recientemente, en el año 2012 se presentó el Síndrome Respiratorio del Medio Oriente (MERS-CoV), patógeno responsable de graves brotes de enfermedades respiratorias que han demostrado la letalidad de los COVID-19 al cruzar la barrera de especies e infectar a los humanos ${ }^{1,9}$.

Según la OMS, el coronavirus presenta un poder de transmisión alto y una letalidad relativamente baja. Esta última aumenta en personas adultas mayores y en personas con patologías crónicas previas (diabetes mellitus, enfermedades cardiovasculares, cerebrovasculares, respiratorias, inmunológicas o personas con obesidad) $)^{14,15}$. Las personas mayores de 70 años presentan una tasa de letalidad del $8 \%$ y los mayores de 80 del $15 \%{ }^{16}$. Dichas tasas son muy superiores a las que presentan niños, jóvenes y adultos, en los cuales la mortalidad no supera el 0,4\%. En general, la tasa de letalidad promedio de COVID-19 es del 2,3\%.

A nivel mundial, hasta el 15 de febrero de 2021 se presentaron 109622245 casos confirmados, 22992942 casos activos, 2417067 fallecidos ${ }^{5,16}$. Los reportes mundiales del COVID-19 son actualizados a diario por la OMS y otras instituciones que presentan a través de mapas el avance de la enfermedad5,16,17. 
Los síntomas principales de los pacientes con coronavirus son fiebre, tos, dificultad para respirar y pérdida del gusto y olfato (anosmia e hiposmia que son síntomas de aparición súbita que es importante evaluar, ya que se pueden presentar en pacientes sin antecedentes de enfermedad nasal previa y son síntomas significativos en relación a la pandemia por COVID-19). Sin embargo, los primeros síntomas en algunos pacientes han sido problemas gastrointestinales; en los casos más severos, la infección puede causar bronquitis o neumonía, síndrome respiratorio agudo severo, fallo o insuficiencia renal e incluso la muerte ${ }^{7,10,18-20}$. De igual forma, países como Reino Unido, Italia, Estados Unidos, Francia, España y Suiza han reportado una posible relación de la enfermedad de Kawasaki con el SARS-CoV-2 en la población infantil ${ }^{21,22}$.

Pese a que han surgido varias propuestas de tratamiento y que algunos países ya comenzaron a implementar la vacuna contra el virus, la pandemia aún sigue en el mundo. Hasta el 15 de febrero de 2021 se reporta un total de 175320450 personas vacunadas $^{16,23}$. A esto se suma el uso de fármacos para tratar la sintomatología $a^{11,20,24}$, como los antivirales, los antiinflamatorios y los anticoagulantes. De igual forma, los estudios para probar la efectividad de otros medicamentos, continúan ${ }^{25}$.

Para la mayoría de personas con un sistema inmunológico no comprometido, será una enfermedad leve y mejorará sin necesidad de ningún cuidado especial. Caso contrario las personas mayores de 65 años con patología previa que tienden a presentar una evolución grave por COVID-19.

El análisis de muestras conjuntivales de casos confirmados y sospechosos de COVID-19 sugieren que la transmisión no se limita al tracto respiratorio y que la exposición del ojo puede proporcionar una forma efectiva para que el virus ingrese al cuer$\mathrm{po}^{2,26,28}$.

Según la clasificación de exposición de trabajadores al COVID-19, la profesión odontológica es una de las que se encuentra en primer lugar por el riesgo de exposición a enfermedades y proximidad física del campo de trabajo ${ }^{29}$. Este riesgo por exposición es muy similar al de otras profesiones donde ocurre la generación de aerosoles, tales como médicos, enfermeras, encargados de laboratorio y, en menor medida pero de considerable riesgo, aquellas profesiones que demandan contacto directo con el público o se desempeñan en espacios reducidos (recepcionistas, cajeros y agencias de atención al cliente) ${ }^{29}$.
Las rutas de transmisión comunes del nuevo coronavirus incluyen transmisión directa de un paciente enfermo a mucosa de ojos, nariz o boca de una persona sana o indirecta por auto inoculación. El odontólogo y su personal auxiliar pueden adquirir por vía directa el coronavirus y generar infecciones, al igual que otros virus, a partir de un paciente enfermo, un portador asintomático o un paciente en recuperación, mediante la exposición directa a los aerosoles que emane por tos y estornudos o los procedimientos operatorios que implican el uso de dispositivos rotatorios de alta velocidad, jeringa triple, cavitrones entre otros, que contribuyen al desplazamiento de los fluidos por el consultorio dental. Y por vía indirecta puede ocurrir al saludar al paciente (manos, codos, puños, mejías, hombros) o tocar con la mano superficies contaminadas por el paciente y llevársela después a la mucosa nasal, oral u ocular2,13-15,23,26,27.

Los cirujanos dentales están propensos a adquirir enfermedades debido al tipo de procedimientos que se realizan dentro de la odontología, ya que esto implica la comunicación cara a cara con los pacientes, la exposición frecuente a saliva, sangre y otros fluidos, así como el manejo de instrumentos afilados $1,11,13,15,23$.

Con el fin de salvaguardar la vida y mantener la calidad de vida de la población salvadoreña, se realizan recomendaciones de acuerdo con el algoritmo de toma de decisiones en clínicas odontológicas en período de emergencia por COVID-19, basadas en la evidencia científica disponible (Figura 1); con el objetivo de realizar la selección de pacientes con necesidades de tratamientos que sean clasificados como urgencias o emergencias. Es importante iniciar con un triaje, de preferencia vía teléfono o videollamada, con el fin de conocer datos sociodemográficos como edad del paciente y descartar la posibilidad que sea parte de un grupo de riesgo. Asimismo, conocer historial de fiebre, goteo nasal, tos seca, diarrea o dificultad para respirar, alteraciones del olfato o del gusto, viajes a países de riesgo, contacto con pacientes positivos a COVID-19, profesión de alto riesgo de contagio por exposición a enfermedades, personal biomédico o voluntarios de hospitales, centros de confinamiento o personas que han estado en contacto estrecho con pacientes que presentan diagnóstico de enfermedad respiratoria aguda o condición sistémica desfavorable.

Esta información contribuirá de manera importante a clasificar al paciente según riesgo de infección y letalidad por COVID-19. El paciente evaluado podrá ser clasificado 
Figura 1. Algoritmo de toma de decisiones en clínicas odontológicas en periodo de emergencia por la COVID-19

\begin{tabular}{|c|c|c|c|c|}
\hline \multicolumn{5}{|c|}{ Triaje por video llamada o teléfono } \\
\hline \multicolumn{5}{|c|}{ 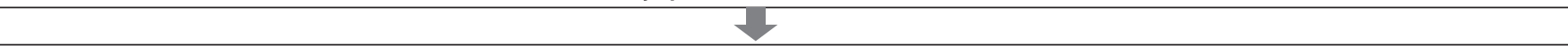 } \\
\hline \multicolumn{5}{|l|}{ 1. ¿Cuál es su edad? } \\
\hline \multicolumn{5}{|c|}{ 2. ¿Tiene alguna condición médica de riesgo de severidad ante la COVID-19 } \\
\hline \multicolumn{5}{|c|}{ 3. ¿Ha tenido fiebre $\left(>37^{\circ} \mathrm{C}\right)$, tos seca, dificultad para respirar, goteo nasal, cansancio o molestias digestivas en los últimos 14 días? } \\
\hline \multicolumn{5}{|c|}{ 4. ¿Presenta o ha presentado alguna alteración en el sentido del olfato o del gusto en los últimos 14 días? } \\
\hline \multicolumn{5}{|c|}{ 5. ¿Ha estado en contacto con personas que presentaron cuadro respiratorio agudo en los últimos 14 días? } \\
\hline \multicolumn{5}{|c|}{ 6. ¿Ha estado en contacto con alguna persona sospechosa o con diagnóstico confirmado de coronavirus? } \\
\hline \multicolumn{5}{|c|}{ 7. ¿Actualmente usted labora en hospitales u otros centros de salud? } \\
\hline \multicolumn{5}{|c|}{ 8. ¿Usted es personal o voluntario en centros de confinamiento en el marco de la pandemia por COVID-19? } \\
\hline \multicolumn{5}{|c|}{ 9. ¿ ¿Pertenece a las profesiones (de salud y con contacto directo al público) con más riesgo de contagio del virus? } \\
\hline \multicolumn{5}{|c|}{ 10. ¿Tiene o ha presentado la enfermedad de la COVID-19? } \\
\hline \multicolumn{5}{|c|}{ 11. En caso de haber presentado la enfermedad, ¿ya cumplió el aislamiento indicado? } \\
\hline \multicolumn{5}{|c|}{ 12. En caso de haber presentado la enfermedad, ¿se realizó una nueva prueba PCR con resultado negativo? } \\
\hline \multicolumn{3}{|c|}{ 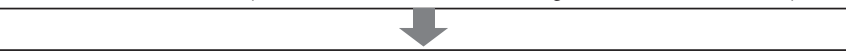 } & \multicolumn{2}{|c|}{$\checkmark$} \\
\hline \multicolumn{3}{|c|}{ Clasificar paciente según riesgo de infección por COVID-19 } & \multicolumn{2}{|c|}{ Clasificar necesidad de tratamiento odontológico } \\
\hline 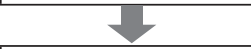 & $\checkmark$ & 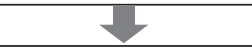 & 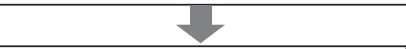 & 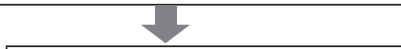 \\
\hline Riesgo bajo & Riesgo moderado* & Riesgo alto* & Tratamiento electivo & Urgencia o emergencia*** \\
\hline$\checkmark$ & $\checkmark$ & $\checkmark$ & 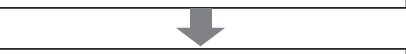 & 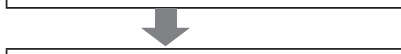 \\
\hline $\begin{array}{l}<60 \text { años (+) } \\
\text { Temperatura }\end{array}$ & $\begin{array}{l}\text { Sí, a cualquier } \\
\text { pregunta. }\end{array}$ & $\begin{array}{l}\text { Sí, a cualquier } \\
\text { pregunta. }\end{array}$ & $\begin{array}{l}\text { Todo aquel no incluido en listado } \\
\text { de emergencias odontológicas** }\end{array}$ & $\begin{array}{c}\text { Todo aquel incluido en listado de } \\
\text { emergencias odontológicas* }\end{array}$ \\
\hline$\left.<37,3^{\circ} \mathrm{C}\right)(+)$ & $<60$ años $(+)$ & $<60$ años $(+)$ & $\checkmark$ & 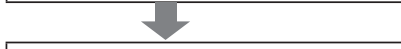 \\
\hline Signos/síntomas (-) & Temperatura & Temperatura & Para los tres niveles de riesgo: & Paciente con riesgo bajo: \\
\hline & $\left.<37,3^{\circ} \mathrm{C}\right)(+)$ & $\left.<37,3^{\circ} \mathrm{C}\right)(+)$ & - Citar, posterior a levanta- & - Resolver emergencia en con- \\
\hline & Signos/síntomas (+) & Signos/síntomas (+) & miento de restricciones por la & sultorio. \\
\hline & & Condición sistémica & COVID-19. & Riesgo moderado: \\
\hline & & destavorable (+) & - Dar indicaciones de autocui- & - Resolver emergencia en con- \\
\hline & & & diagnóstico establecido. & Riesgo alto: \\
\hline $\begin{array}{l}{ }^{*} \text { Ante cualquier sosp } \\
\text { médica para evaluaci } \\
{ }^{* *} \text { Todo tratamiento o } \\
{ }^{* *} \text { Consultar: Facultc } \\
\text { en odontología. } \\
\text { Enlaces: https://pesqu }\end{array}$ & $\begin{array}{l}\text { de paciente infectad } \\
\text { nergencia deberá ser } \\
\text { Odontología, univer } \\
\text { ovsalud.org/portal/re }\end{array}$ & $\begin{array}{l}\text { sponer tratamiento de } \\
\text { zado bajo consentimic } \\
\text { d de El Salvador 2020; } \\
\text { ce/pt/biblio-1147752 }\end{array}$ & $\begin{array}{l}\text { ncia y remitir a interconsulta } \\
\text { nformado del paciente. } \\
\text { gencias odontológicas frecuentes }\end{array}$ & $\begin{array}{l}\text { - Resolver emergencia por video } \\
\text { Ilamada o teléfono con farma- } \\
\text { coterapia según diagnóstico } \\
\text { establecido o remitir a atención } \\
\text { hospitalaria ante riesgo de } \\
\text { septicemía o hemorragia..*** }\end{array}$ \\
\hline
\end{tabular}
biblioref/2021/02/1147752/vf-19022021-emergencias-frecuentes-en-odontologia.pdf

****Recomendable bajo sedación para trabajar en ambiente controlado.

en riesgo bajo, moderado o alto. En el grupo de los pacientes catalogados con riesgo bajo se incluyen las personas menores de 60 años, con temperatura menor a $37,3^{\circ} \mathrm{C}$, signos y síntomas negativos. En el grupo de los pacientes con riesgo moderado, se encuentran aquellas personas que respondan «sí» a cualquier pregunta realizada en el triaje (Figura 1), personas menores de 60 años, con temperatura mayor o igual a $37,3^{\circ} \mathrm{C}$, signos y síntomas positivos a COVID-19. Mientras que, los pacientes con riesgo alto son aquellos que afirmen a cualquier pregunta realizada en el triaje (Figura 1), además de pertenecer al grupo etario de riesgo (personas de más de 60 años), presentar temperatura mayor de $37.3^{\circ} \mathrm{C}$ con signos, síntomas y enfermedad sistémica desfavorable previa.

De acuerdo con la clasificación de riesgo de infección del paciente, se evaluará la necesidad de tratamiento odontológico, como tratamiento electivo y de urgencia o emergencia. Por lo que, en pacientes con demanda de tratamiento electivo, se deberá posponer la atención y citar en un período posterior al levantamiento de medidas y restricciones nacionales ${ }^{6}$; en estos casos, se debe brindar indicaciones de autocuidado o farmacoterapia con base en el diagnóstico establecido. A diferencia de los pacientes clasificados para tratamientos urgentes y emergentes, que debido al riesgo vital identificado recibirán la atención odontológica requerida. Por lo que todo tratamiento debe ser atendido bajo consentimiento o asentimiento informado específico para cada emergencia. Los pacientes del nivel de riesgo bajo que se presenten con una urgencia o emergencia a la consulta dental o hayan sido evaluados previamente, se deberá resolver la necesidad de tratamiento dentro del consultorio dental, tomando todas las medidas de bioseguridad $^{12}$. Los pacientes clasificados en un nivel de riesgo moderado, 
deberán ser remitidos a interconsulta médica para descartar una posible infección por COVID-19; al obtener un resultado negativo se podrá resolver la emergencia en el consultorio dental. Mientras que a los pacientes con riesgo alto de infección se deberá resolver la emergencia por videollamada o por teléfono con farmacoterapia en primera instancia, con base en el diagnóstico oral establecido. Ante el riesgo de septicemia o hemorragia, se deberá remitir al paciente para atención hospitalaria. Se recomienda trabajar en un ambiente controlado y de preferencia bajo sedación.

Al igual que otros virus, el SARS-CoV-2 demanda el seguimiento riguroso de los protocolos de bioseguridad para la protección del personal odontológico y pacientes, por lo que se debe continuar con la práctica permanente de estos protocolos para evitar infecciones cruzadas o contagios dentro del consultorio dental. Todos los procedimientos de emergencia deben limitarse a lo estrictamente indispensable a fin de acatar las disposiciones brindadas por las autoridades de salud. Se recomienda limpiar y desinfectar los objetos y las superficies, utilizando solución de Dakin al 0,5 \% sustancia conocida por hipoclorito de sodio $(\mathrm{NaClO})$. Es recomendado a dicha concentración por ser un agente antiséptico, de baja toxicidad y eficaz contra un amplio espectro de virus, hongos, esporas, bacterias aeróbicas y anaeróbicas ${ }^{26,30}$. Esta solución desinfectante debe ser preparada a partir de una solución principal y el cambio deberá ser a diario. Posteriormente, limpiar con alcohol isopropílico al $70 \%$ o alcohol etílico para inactivar el hipoclorito y evitar la corrosión de superficies de trabajo metálicas.

Se recomienda el empleo de medidas de bioseguridad sin excepciones como el uso de guantes, respiradores de careta filtrante N95 con certificación del Instituto Nacional de Seguridad y Salud Ocupacional (NIOSH, por sus siglas en inglés), máscaras estándar FFP2 establecidas por la Unión Europea o las FFP3, protección ocular (lentes o careta protectora), gorro de tipo oruga, gabacha manga larga o gabachón descartable, zapateras desechables y una ventilación adecuada del consultorio dental14,15. Se debe tomar en cuenta el uso de alcohol gel al $70 \%$ durante la colocación y el retiro correcto del equipo de protección para evitar contaminaciones.

Para evitar posibles contagios por contacto indirecto al tocar con la mano objetos contaminados y tocar después la mucosa oral, nasal u ocular; se recomienda retirar periódicos, revistas, juguetes u otros objetos de contacto frecuente de las salas de espe$r^{29-31}$. Además, realizar lavado de manos antes y después de la atención de cada paciente y antes de la colocación de los guantes. Primero lavar con agua y jabón, secar con papel toalla y luego frotarse las palmas y los dedos con alcohol gel, durante 20-30 segundos.

El uso de enjuague bucal antimicrobiano preoperatorio podría reducir la carga viral en cavidad oral. Para tal efecto, la literatura recomienda el uso de povidona yodada al 0,2\% o peróxido de hidrógeno al 1,0 \% durante 1 minuto; ya que se ha reportado que el SARS y el MERS son susceptibles a la povidona y el SARS-CoV-2 ha mostrado vulnerabilidad a la oxidación por el peróxido de hidógeno². El cloruro de N-hexadecilpiridinio o cloruro de cetilpiridinio (CPC), es un compuesto catiónico de amonio cuaternario que no es oxidante ni corrosivo, ha demostrado su eficacia como agente inhibidor de amplio espectro de coronavirus al ser el noveno fármaco de 36 sustancias probadas ante 4 virus incluyendo el MERS-CoV; asimismo, es un agente antiséptico, antibacteriano, antiplaca y antigingivitis; por otra parte, se ha sugerido el uso de la Clorhexidina para reducir la transmisión viral, por sus propiedades antisépticas, antibacterianas, antigingivitis, biguanidas, virus y levaduras; sin embargo, la evidencia contra el coronavirus humano con envoltura, aún no es concluyente ${ }^{32}$.

Finalmente, las atenciones de urgencias o emergencias odontológicas deberán ser programadas por citas espaciadas para evitar aglomeraciones y bajo la aplicación estricta de los protocolos de desinfección. Para los casos en los que la pieza de mano sea indispensable su uso, se recomienda la utilización de piezas de mano dental con válvulas antirretractivas $u$ otros diseños antirreflujo, ya que puede reducir significativamente el retorno de la carga viral a través de las mangueras de la pieza de mano.

\section{Conclusión}

El algoritmo de toma de decisiones en clínicas odontológicas durante el período de emergencia por COVID-19, debe constituirse en una recomendación a ser puesta en práctica por el odontólogo que atienda pacientes en estado de emergencia. Por cuanto permite clasificar el riesgo de infección y letalidad, precisando los tratamientos en electivos, de urgencia o emergencia.

\section{Referencias bibliográficas}

1. Zhu N, Zhang D, Wang W, Li X, Yang B, Song $J$, et al. A novel coronavirus from patients with pneumonia in China, 2019. N Engl J 
Med. 2020;382(8):727-33. DOl: http://www. nejm.org/doi/10.1056/NEJMoa2001017

2. Peng X, Xu X, Li Y, Cheng L, Zhou X, Ren B. Transmission routes of 2019-nCoV and controls in dental practice. Int J Oral Sci. 2020;12(1):1-6. DOI: https://doi. org/10.1038/s41368-020-0075-9

3. Organización Panamericana de la Salud. La OMS declara que el nuevo brote de coronavirus es una emergencia de salud pública de importancia internacional. PAHO, 2020. Fecha de consulta: 21 de abril de 2020. Disponible en: https://www. paho.org/hq/index.php?option=com co ntent\&view=article\&id $=15706$ :statem ent-on-the-second-meeting-of-theinternational-health-regulations-2005emergency-committee-regarding-theoutbreak-of-novel-coronavirus-2019ncov\&ltemid $=1926 \&$ lang $=$ en

4. Organización Mundial de la Salud. Alocución de apertura del director general de la OMS en la rueda de prensa sobre la COVID-19 celebrada el 11 de marzo de 2020. OMS, 2020. Fecha de consulta: 29 de marzo de 2020. Disponible en: https://www.who. int/es/dg/speeches/detail/who-directorgeneral-s-opening-remarks-at-the-mediabriefing-on-covid-19---11-march-2020

5. Universidad Johns Hopkins. An interactive web-based dashboard to track COVID-19 in real time. Lancet. 2020;20(5):533-4. DOI: https://doi.org/10.1016/S14733099(20)30120-1

6. López A, Domínguez R. Medidas de contingencia ante el COVID-19 en El Salvador, Alerta. 2021;4(1):78-79. DOI 10.5377/alerta.v4i1.10762

7. Ministerio de Salud. Situación nacional COVID-19. 2020. Fecha de consulta: 18 de abril de 2020. Disponible en: $\underline{\text { https:// }}$ covid19.gob.sv/

8. Cheng ZJ, Shan J. 2019 Novel coronavirus: where we are and what we know. Infection 48, 155-163 (2020). https://doi.org/10.1007/ s15010-020-01401-y

9. Schoeman D, Fielding BC. Coronavirus envelope protein: Current knowledge. Virol J. 2019;16(69):1-22. DOI: https://doi. org/10.1186/s12985-019-1182-0

10. Guan W, Ni Z, Hu Y, Liang W, Ou C, He J, et al. Clinical Characteristics of Coronavirus Disease 2019 in China. N Engl J Med. 2020;382(18):1708-20. DOI: http://www. nejm.org/doi/10.1056/NEJMoa2002032

11. Yu F, Du L, Ojcius DM, Pan C, Jiang S. Measures for diagnosing and treating infections by a novel coronavirus responsible for a pneumonia outbreak originating in Wuhan, China. Microbes Infect. 2020;22(2):74-9. DOI: https://doi. org/10.1016/j.micinf.2020.01.003
12. Ge ZY, Yang LM, Xia JJ, Fu XH, Zhang YZ. Possible aerosol transmission of COVID-19 and special precautions in dentistry. J Zhejiang Univ Sci B. 2020;21 (R780.1; R511):1-8. DOI: https://doi.org/10.1631/jzus. B2010010

13. Spagnuolo G, Vito D De, Rengo S, Tatullo M. COVID-19 Outbreak: An Overview on Dentistry. Int J Environ Res Public Heal. 2020;17(6 PG):2094. DOl: https://doi. org/10.3390/ijerph17062094

14. Jin YH, Cai L, Cheng ZS, Cheng H, Deng T, Fan YP, et al. A rapid advice guideline for the diagnosis and treatment of 2019 novel coronavirus (2019-nCoV) infected pneumonia (standard version). Mil Med Res. 2020;7(1):4. DOI: https://doi.org/10.1186/ $\underline{\text { s40779-020-0233-6 }}$

15. Meng L, Hua F, Bian Z. Coronavirus Disease 2019 (COVID-19): Emerging and Future Challenges for Dental and Oral Medicine. J Dent Res. 2020;99(5):481-7. DOI: https://doi. org/10.1177/0022034520914246

16. Mamoon N, Rasskin G. COVID-19 Visualizer. Universidad de Carnegie mellon. 2020. Fecha de consulta: 18 de abril de 2020. Disponible en: https://www.covidvisualizer. com/

17. World Health Organization. WHO COVID-19 Dashboard. World Health Organization. 2020. Fecha de consulta: 17 de abirl de 2020. Disponible en: https://who.sprinklr.com/

18. Keyes M, Thibodeau R. Dakin Solution (Sodium Hypochlorite). StatPearls; 2018. Fecha de consulta: 19 de marzo de 2020. Disponible en: http://www.ncbi.nlm.nih. gov/pubmed/29939693

19. Cao B, Wang Y, Wen D, Liu W, Wang J, Fan $\mathrm{G}$, et al. A Trial of Lopinavir-Ritonavir in Adults Hospitalized with Severe Covid-19. N Engl J Med. 2020; 382(19): 1787-1799. DOI: https://www.nejm.org/doi/full/10.1056/ NEJMoa2001282

20. Holshue ML, DeBolt C, Lindquist S, Lofy $\mathrm{KH}$, Wiesman J, Bruce H, et al. First case of 2019 novel coronavirus in the United States. N Engl J Med. 2020;382(10):929-36. DOI: http://www.nejm.org/doi/10.1056/ NEJMoa2001191

21. Vázquez C. Romero I, Huertas M, Fernández C, Pignatelli R. Alerta sobre asociación COVID 19 y probable Enfermedad de Kawasaki en pacientes. Sociedad Interamericana de Cardiología. Fecha de publicación: 10 de mayo de 2020. 5 p. Disponible en: http://www.siacardio. com/novedades/covid-19/alertasobre-asociacion-covid-19-y-probableenfermedad-de-kawasaki-en-pacientespediatricos/

22. Verdoni L, Mazza A, Gervasoni A, Martelli L, Ruggeri M, Ciuffreda M, et al. An outbreak of severe Kawasaki-like disease at the Italian 
epicentre of the SARS-CoV-2 epidemic: an observational cohort study. The Lancet. 2020;395(10239): 1771-1778. DOl: https:// doi.org/10.1016/S0140-6736

23. Sabino-Silva R, Jardim A, Siqueira WL. Coronavirus COVID-19 impacts to dentistry and potential salivary diagnosis. Clin Oral Investig. 2020; 24:1619-21. DOI: https://doi. org/10.1007/s00784-020-03248-x

24. Rubin EJ, Baden LR, Morrissey S. Audio Interview: New Research on Possible Treatments for Covid-19. N Engl J Med. 2020;382(12):e30. DOI: 10.1056/ NEJMe2005759

25. The Lancet Hematology. COVID-19 coagulopathy: an evolving story. The Lancet. 2020;7(6):E425. DOl: https://doi.org/10.1016/ $\underline{\mathrm{S} 2352-3026(20) 30151-4}$

26. Kohn WG. Cover story guidelines for infection control in dental health care settings-2003. J Am Dent Assoc. 2004;135(1):33-47. DOI: https://doi. org/10.14219/jada.archive.2004.0019

27. Asociación Dental Americana. 2019 Novel Coronavirus. ADA. 2020;4. Disponible en: https://www.cdc.gov/flu/professionals/ infectioncontrol/healthcaresettings.htm

28. Azhar El, El-Kafrawy SA, Farraj SA, Hassan AM, Al-Saeed MS, Hashem AM, et al. Evidence for Camel-to-Human Transmission of MERS Coronavirus. n engl j med. 2014;26:2499_ 505. DOI: 10.1056/NEJMoa1401505
29. Gamio L. The Workers Who Face the Greatest Coronavirus Risk. The New York Times. 2020.

Fecha de consulta: 22 de abirl de 2020. Disponible en: https://www.nytimes.com/ interactive/2020/03/15/business/economy/ coronavirus-worker-risk.html?action=click\& module $=$ Top + Stories\&pgtype $=$ Homepage

30. Antunovic FA, Fernández CA, Aranda EE, Ale VS, Marecos MC, et al. La solución de Dakin-Carrel. Flebología y Linfología. 2013;8(20):1230-5. Disponible en: https://silo. tips/download/la-solucion-de-dakin-carrel

31. Centros de Prevención y Contro de Enfermedades. Guía para la prevención de infecciones en entornos ambulatorios: expectativas mínimas para una atención segura. 2017. 44 p. Disponible en: $\underline{\text { http:// }}$ www.cdc.gov/hai/settings/outpatient/ outpatient-care-guidelines.html

32. Herrera D, Serrano J, Roldán S. et al. Is the oral cavity relevant in SARS-CoV-2 pandemic?. Clin Oral Invest. 2020;24,29252930. DOI: https://doi.org/10.1007/s00784020-03413-2 